\title{
Invariant Mean Electrical Axis in Electrocardiogram
}

\author{
Kjell Le ${ }^{1}$, Trygve Eftestø $1^{1}$, Kjersti Engan $^{1}, \varnothing y u n n$ Kleiven ${ }^{2}$, Stein $\emptyset \mathrm{rn}^{1,2}$ \\ ${ }^{1}$ Department of Electrical Engineering, University of Stavanger, Stavanger, Norway \\ ${ }^{2}$ Department of Cardiology, Stavanger University Hospital, Stavanger, Norway
}

\begin{abstract}
The mean electrical axis (MEA) is is an important measurement in clinical assessment. However, currently there is no agreement on a standardized calculation of the MEA. In order to standardize the MEA calculation, the following issues need to be addressed: 1) The net direction or net potential in the measurement of MEA needs to be defined. 2) Which underlying heart model which describes the electrical activity of the heart to use. 3) Which electrocardiogram (ECG) leads to calculate MEA from.

In present use for some applications, e.g. ECG-derived respiration, lead pairs I and III or I and aVF are used in axis calculation without further consideration of scaling and angle between the leads. This may cause an error up to $30^{\circ}$ in the Einthoven model.

The aim of this work is to derive correct systematic equations for determining the heart vector (dipole) and MEA from any pair of leads in the frontal plane. Since any pair of leads can define the frontal plane, it is more robust to calculate the MEA from each lead pair and take the mean of the results. From the results two different net potential definitions, $V_{\text {area }}$ and $V_{\text {sum }}$, gave approximately the same answer.
\end{abstract}

\section{Introduction}

The mean electrical axis (MEA) is the net direction (an angle) of the heart vector, dipole, [1] during depolarization and polarization processes. MEA is important because it correlates with the heart orientation, which is useful in clinical assessment [2]. MEA might not be specified, but often it refers to the net direction of the QRS complex [3-5] in the frontal plane, and not the actual direction in 3D space, in this case, of the ventricular depolarization.

Even though there is an ongoing standardization and interpretation of electrocardiogram (ECG) [6,7], there is still no clearly defined and correct method to find the MEA. The discrepancies can be divided into 3 parts.

The first issue addresses the definition of net direction of the heart vector. The direction of a dipole is determined from the voltages in the leads thus net direction can be translated to net potentials in the leads. One approach is to find the net potential as the sum of the dominant QRS wave forms $\left(V_{r s}\right)[4,7]$.Another approach is to take the whole depolarization process into consideration, or a segment of it $[2,8-12]$, where the net potential is the area under the curve $\left(V_{\text {area }}\right)$ or sum of voltages of the segment $\left(V_{\text {sum }}\right)$.

The second issue addresses the heart model, and how to find the dipole. In the classical homogeneous conducting sphere with a centric dipole model the voltage in the limb leads are dot products between the heart vector and the lead axes (unit vector) [6]. The leads form the Einthoven's triangle, together with the augmented limb leads, define the hexaxial reference system (figure 1). The Einthoven's triangle is in the frontal plane. However, in other models such as Burge and Frank the triangle formed by the limb leads are not in the frontal plane [13]. For these models it is possible to project the dipole to the plane spanned by the lead vectors (triangle plane) and then project the dipole to the frontal plane to find the MEA. This induce an error because of the projection to the triangle plane first. Thus, it can be thought that it would be more correct to find the dipole, and thereafter project the dipole to the frontal plane. Methods to find the dipole directly from 12-leads ECG is to use inverse Dower- or Kors transform [14,15].

The third issue addresses the linear dependency between the limb leads and augmented limb leads. Ideally any combination of 2 of these leads will result in the same axis calculation. However, after preprocessing to enhance the signal quality, the results might vary. This variation can be used as an indirect measurement of fidelity.

In present use for some applications, e.g. ECG-derived respiration $[10,11]$, lead pairs I and III or I and aVF are used in axis calculation without further consideration of scaling, e.g. the lead vectors of augmented limb leads are not unit vectors compared to limb leads (figure 1), and angle between the leads with the Einthoven model. This can be considered a crude approximation, and may cause an error up to $30^{\circ}$. Geometrically derived formulae exist [16], but are inconvenient in usage. The aim of this work is to derive correct systematic equations for determining the dipole and MEA from any pair of leads in the hexaxial reference system. Since any pair of leads can be used to find 
the MEA, it is more robust to calculate the MEA from each lead pair and take the mean of the results. Comparison will be made between different definitions of net potential, $V_{r s}, V_{\text {area }}$ and $V_{\text {sum }}$, and with reference axis calculations done by the electrocardiograph (Welch Allyn).

\section{Dataset}

The dataset is from the North Sea Race Endurance Exercise Study (NEEDED) with ClinicalTrials.gov identifier: NCT02166216. Each ECG record has a duration of $10 \mathrm{~s}$ with a sampling frequency of $f_{s}=600 \mathrm{~Hz}$. The number of records are approximately 3000.

\section{Method}

\subsection{Mean Electrical Axis}

Table 1. Lead vectors, AHA coordinate system.

\begin{tabular}{ccccccc} 
Lead & I & II & III & aVL & -aVR & aVF \\
\hline $\mathrm{x}$ & 1 & 0.5 & -0.5 & 0.75 & 0.75 & 0 \\
$\mathrm{y}$ & 0 & $\sqrt{3} / 2$ & $\sqrt{3} / 2$ & $-\sqrt{3} / 4$ & $\sqrt{3} / 4$ & $\sqrt{3} / 2$
\end{tabular}

In this subsection an equation set to find MEA is derived for the Einthoven model. For a matrix $\boldsymbol{A}, \boldsymbol{A}^{+}, \operatorname{col}(\boldsymbol{A})$, row $(\boldsymbol{A})$ denote the pseudoinverse, column space of $\boldsymbol{A}$, and row space of $\boldsymbol{A}$, respectively. $\langle\cdot, \cdot\rangle$ denotes the inner product.

To find the MEA in the frontal plane from the limb leads, an orthogonal projection $\underline{\hat{d}}$ of the dipole $\underline{d}$ onto plane $\boldsymbol{W}$ spanned by the lead vectors is first found. Thereafter an orthogonal projection of $\hat{d}$ onto the $x y$ ( frontal) plane from $\boldsymbol{W}$ finds the MEA in the frontal plane. Since the lead vectors only span the plane $\boldsymbol{W}$ an error from the orthogonal complement of $\boldsymbol{W}$ will occur. To minimize this error an extra lead which are not in $\boldsymbol{W}$ must be used to find the dipole $d$.

Let $\underline{d}=\left(d_{x}, d_{y}, d_{z}\right)^{T}$ be a dipole vector at time $t$. The voltage in a lead $i$ at time $t$ is the inner product of $\underline{d}$ with the lead vector $\underline{v}_{i}=\left(x_{i}, y_{i}, z_{i}\right)^{T}$ is given as

$$
V_{i}=\left\langle\underline{v}_{i}, \underline{d}\right\rangle=\underline{v}_{i}^{T} \underline{d} .
$$

The lead vector $\underline{v}_{i}$ is not necessarily a unit vector. From the Einthoven, Burger and Frank's triangles the only certainty is the direction of the lead vectors [13]. However, the scaling does not affect the direction of the dipole. Let

$$
\boldsymbol{A}=\left[\underline{v}_{i}, \underline{v}_{j}\right]^{T}, \quad \underline{b}=\left[V_{i}, V_{j}\right]^{T}
$$

where $i \neq j$, then a matrix equation of the form

$$
\boldsymbol{A} \underline{d}=\underline{b}
$$

can be set up. $\boldsymbol{A}$ has rank $r=2$ and does not have full column rank. Let $\boldsymbol{A}=\boldsymbol{U} \boldsymbol{D} \boldsymbol{V}^{T}$ be the reduced singular value decomposition [17]. The columns in $\boldsymbol{U}$ form an orthonormal basis set for $\operatorname{col}(\boldsymbol{A})$ and the columns in $\boldsymbol{V}$ form an orthonormal basis set for $\operatorname{row}(\boldsymbol{A}) . \boldsymbol{D}$ is a diagonal matrix where the entries are non-zero singular values. The pseudoinverse is defined to be

$$
\boldsymbol{A}^{+}=\boldsymbol{V} \boldsymbol{D}^{-1} \boldsymbol{U}^{T}
$$

The least square solution of the matrix equation is

$$
\begin{aligned}
\hat{\hat{d}} & =\boldsymbol{A}^{+} \underline{b} \\
& =\left(\boldsymbol{V} \boldsymbol{D}^{-1} \boldsymbol{U}^{T}\right)\left(\boldsymbol{U} \boldsymbol{D} \boldsymbol{V}^{T} \underline{d}\right)=\boldsymbol{V} \boldsymbol{V}^{T} \underline{d} .
\end{aligned}
$$

Hence $\underline{\hat{d}}$ is the orthogonal projection of $\underline{d}$ onto the plane $\boldsymbol{W}$. Because of linearity, from eq. (5) it can be seen that the sum of the dipole vectors (at different points in time, $k$ ) can be determined from the sum of ECG voltages:

$$
\sum_{k} \underline{\hat{d}}_{k}=\boldsymbol{A}^{+}\left(\sum_{k} \underline{b}_{k}\right) .
$$

If the plane $\boldsymbol{W}$ is the frontal plane then it is now possible to find the MEA as an angle

$$
\beta=\arctan \left(\frac{\hat{d_{y}}}{\hat{d_{x}}}\right),
$$

where $\hat{d}_{x}$ and $\hat{d}_{y}$ is the $x$ and $y$ component of the resulting dipole in (7). The net potential is the sum of voltages in (7).

\subsection{Net Potential}

A method to calculate the MEA is to use a net potential that is the sum of peak voltages of the $R$ and $S$ waves, although it is much simpler to use the max and min value of the QRS complex. Let $x(n)$ be an ECG-segment which includes the QRS complex then the net potential can be express as

$$
V_{r s}=\max (x(n), 0)+\min (x(n), 0)
$$

An alternative to using max and min value of the QRS complex is to use the area under the curve (AUC). If the trapezoid method is used to estimate the AUC, it can be shown that:

$$
V_{\text {area }}=x(0) / 2+\sum_{n=1}^{N-2} x(n)+x(N-1) / 2
$$

where $N$ is the length of the segment $x(n)$. However, from eq. (7) the sum of heart vectors corresponds to the sum of voltages, giving the alternative definition of net potential as

$$
V_{\text {sum }}=\sum_{n=0}^{N-1} x(n)
$$




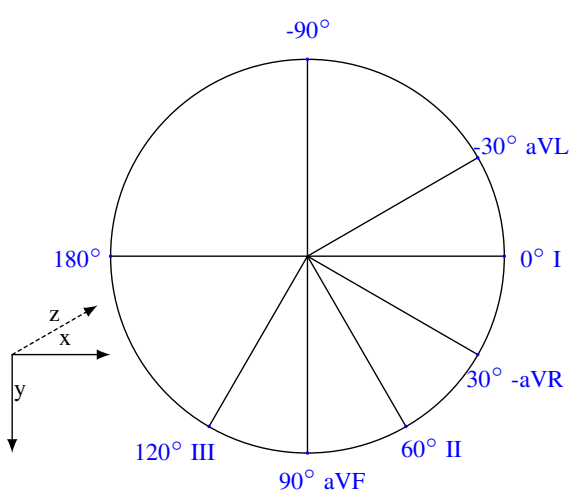

Figure 1. Hexaxial reference system.

\subsection{Preprocessing}

The ECG signals are preprocessed to remove baseline wander, high frequency above $150 \mathrm{~Hz}$ [6] and powerline interference. Thereafter signal averaging $[18,19]$ is performed to find the most common QRS template.

For a successful signal averaging it is necessary to synchronize the signal segments during clustering. The average square difference function (ASDF) method [20] is used to synchronize the segments. The main benefit of this method is perfect synchronization in the absent of noise. The spectrum is also not needed when compared to generalized correlation methods in [21].

Finally an isoelectric (asystole) correction is used to align PQ segments close to $0 \mathrm{~V}$.

\subsection{Assessment}

Let a model $G=\left\{g_{i, j}\right\}=\left[\underline{g}_{1}, \underline{g}_{2}, \ldots, \underline{g}_{L}\right]$ be a matrix of size $(K, L)$ such that each element corresponds to a MEA. Each column denotes an ECG record and each row denotes a lead pair.

Column deviation for column $\underline{g}_{j}$ is defined to be

$$
D_{c, j}=\sqrt{\frac{1}{K} \sum_{i=1}^{K}\left(g_{i, j}-R_{i, j}\right)^{2}}
$$

where $R_{i, j}$ represent a reference MEA $\left(R_{r e f}\right)$, a different model's MEA, or a central value such as the mean, $\mathrm{E}\left\{\underline{g}_{j}\right\}$. When $R_{i, j}=\mathrm{E}\left\{\underline{g}_{j}\right\}$ then the column deviation is the standard deviation between lead pairs. Expected column deviation is denoted as

$$
M_{c}=\frac{1}{L} \sum_{j=1}^{L} D_{c, j}
$$

The column deviation is a measure of dispersity throughout the lead pair in a record. The expected column deviation is the mean value of the column deviation. A low value indicate a good conformity between central value or reference MEA with the lead pairs' MEA.

Row deviation for a row $i$ is defined to be

$$
D_{r, i}=\sqrt{\frac{1}{L} \sum_{j=1}^{L}\left(g_{i, j}-R_{i, j}\right)^{2}} .
$$

The row deviation is a measure of how much each lead pair differ from a true value $R_{i, j}$.

\section{Results}

The percentage of ECGs with standard deviation with less than $15^{\circ}$ between the lead pairs are $94.8 \%$ for $V_{r s}$, $98.8 \%$ for $V_{\text {area }}$ and $98.7 \%$ for $V_{\text {sum }}$. Table 2 shows the lead pair deviation (row deviation). Table 3 shows the expected column deviation for the different choices of reference $R_{i, j}$. Table 4 shows number of records within each axis deviation category according to axis deviation criteria defined in [7]. Missing calculations from the electrocardiograph is added to the undefined category.

Table 2. Row deviation for the three net potential definitions, $V_{r s}, V_{\text {area }}$ and $V_{\text {sum }}$, with all combinations of lead pairs. Bold face shows top 3 most deviated lead pairs. $\left(\right.$ Degree,$\left.^{\circ}\right)$

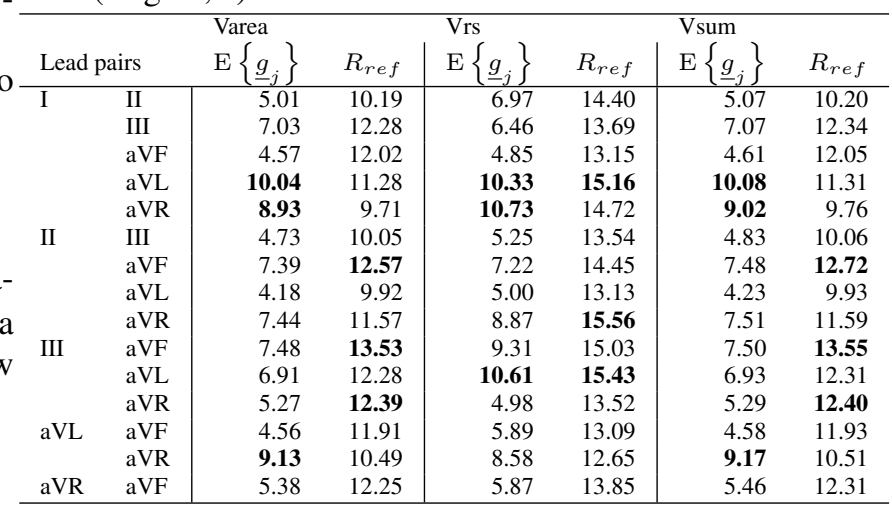

Table 3. Expected column deviation for different choices of reference $R_{i, j}$. (Degree, ${ }^{\circ}$ )

\begin{tabular}{lrrr}
\hline & \multicolumn{3}{c}{$R_{i, j}$} \\
& Varea & $\mathrm{E}\left\{\begin{array}{c}g \\
g\end{array}\right\}$ & $R_{\text {ref }}$ \\
\hline Varea & 0.00 & 3.27 & 4.54 \\
Vrs & 9.60 & 5.81 & 9.75 \\
Vsum & 0.08 & 3.29 & 4.57 \\
\hline
\end{tabular}

Table 4. Number of records within each axis deviation category from mean, $R_{i, j}=\mathrm{E}\left\{\underline{g}_{j}\right\}$, lead pairs.

\begin{tabular}{lrrrr}
\hline & Vrs & Varea & Vsum & ref. \\
\hline Left axis deviation & 60 & 113 & 113 & 107 \\
Right axis deviation & 43 & 80 & 80 & 59 \\
Normal & 2908 & 2814 & 2814 & 2841 \\
Undefine & 1 & 5 & 5 & 11 \\
\hline
\end{tabular}




\section{Discussion and Conclusion}

The results show that the two net potentials $V_{\text {area }}$ and $V_{\text {sum }}$ are quite similar. Overall results show that these two net potentials have the smallest deviation, such as the percentage of ECGs with less than $15 \%$ standard deviation between the leads is higher for $V_{\text {area }}(98.8 \%)$ and $V_{\text {sum }}(98.7 \%)$ compared to $V_{r s}(94.8 \%)$. Table 2 shows that lead pair I-aVL, I-aVR, III-aVL, and aVR-aVL are amongst lead pairs which differ most from the mean. There is a slight difference between $V_{\text {area }}$ and $V_{\text {sum }}$ which is shown in table 3. The first column shows deviation between $V_{\text {area }}$ and $V_{\text {sum }}$ to be $0.08^{\circ}$. The deviation between $V_{\text {area }}$ and $V_{r s}$ is $9.60^{\circ}$. Table 3 also shows that $V_{\text {area }}$ has the smallest deviation between lead pairs with respect to the reference MEA. Table 4 shows the number of records in different axis deviation categories changes depending in which method is used. Because of this disparity between the models a standard is highly sought otherwise it might lead to erroneous clinical conclusions, e.g. normal axis deviation is categorized as left axis deviation. Since the MEA can be calculated from any pair of leads in the frontal plane, it is more robust to calculate the MEA from each pair and take the mean of the results. $V_{\text {area }}$ and $V_{\text {sum }}$ give less variability between the leads and should be applied to ensure fidelity in clinical use.

\section{Acknowledgements}

This work is part of the North Sea Race Endurance Exercise Study (NEEDED) in Norway.

\section{References}

[1] Burger H, Van Milaan J. Heart-vector and leads.: Part III geometrical representation. British Heart Journal 1948; 10(4):229.

[2] de Luna AB. Clinical Electrocardiography: A Textbook. John Wiley \& Sons, 2012.

[3] Goldberger AL, Goldberger ZD, Shvilkin A. Clinical Electrocardiography: A Simplified Approach E-Book. Elsevier Health Sciences, 2017.

[4] Hall JE. Guyton and Hall textbook of medical physiology e-Book. Elsevier Health Sciences, 2015.

[5] Gacek A, Pedrycz W. ECG signal processing, classification and interpretation: a comprehensive framework of computational intelligence. Springer Science \& Business Media, 2011.

[6] Kligfield P, Gettes LS, Bailey JJ, Childers R, Deal BJ, Hancock EW, van Herpen G, Kors JA, Macfarlane P, Mirvis DM, Pahlm O, Rautaharju P, Wagner GS. Recommendations for the standardization and interpretation of the electrocardiogram, part I: The electrocardiogram and its technology. Circulation 2007;115(10):1306-1324.

[7] Surawicz B, Childers R, Deal BJ, Gettes LS. AHA/ACCF/HRS recommendations for the standard- ization and interpretation of the electrocardiogram, part III: Intraventricular conduction disturbances. Circulation 2009; 119(10):e235-e240.

[8] Crawford J, Doherty L. Practical aspects of ECG recording. M\&K Update Ltd, 2012.

[9] Zhao L, Reisman S, Findley T. Derivation of respiration from electrocardiogram during heart rate variability studies. In Computers in Cardiology 1994. Sep 1994; 53-56.

[10] Behbehani K, Vijendra S, Burk J, Lucas E. An investigation of the mean electrical axis angle and respiration during sleep. In 24th Annual Conference and the Annual Fall Meeting of the Biomedical Engineering Society EMBS/BMES Conference, 2002., volume 2. IEEE, 2002; $1550-1551$.

[11] Park SB, Noh YS, Park SJ, Yoon HR. An improved algorithm for respiration signal extraction from electrocardiogram measured by conductive textile electrodes using instantaneous frequency estimation. Medical Biological Engineering Computing 2008;46(2):147-158.

[12] Bailon R, Sornmo L, Laguna P. A robust method for ECGbased estimation of the respiratory frequency during stress testing. IEEE Transactions on Biomedical Engineering July 2006;53(7):1273-1285.

[13] Malmivuo P, Malmivuo J, Plonsey R. Bioelectromagnetism: principles and applications of bioelectric and biomagnetic fields. Oxford University Press, USA, 1995.

[14] Guillem MS, Sahakian AV, Swiryn S. Derivation of orthogonal leads from the 12-lead electrocardiogram. Performance of an atrial-based transform for the derivation of $\mathrm{P}$ loops. Journal of Electrocardiology 2008;41(1):19-25.

[15] Kors J, Van Herpen G, Sittig A, Van Bemmel J. Reconstruction of the Frank vectorcardiogram from standard electrocardiographic leads: diagnostic comparison of different methods. European Heart Journal 1990;11(12):1083-1092.

[16] Dubin S, Staib J. Numerical calculation of the mean electrical axis of electrocardiographic deflections. Journal of Electrocardiology 1977;10(1):77-78.

[17] Lay D. Linear Algebra and Its Applications. AddisonWesley, 2012. ISBN 9780321385178.

[18] Sörnmo L, Laguna P. Bioelectrical signal processing in cardiac and neurological applications, volume 8. Academic Press, 2005.

[19] Goldberger JJ, Ng J. Practical signal and image processing in clinical cardiology. Springer, 2010.

[20] Jacovitti G, Scarano G. Discrete time techniques for time delay estimation. IEEE Transactions on Signal Processing 1993;41(2):525-533.

[21] Knapp C, Carter G. The generalized correlation method for estimation of time delay. IEEE Transactions on Acoustics Speech and Signal Processing 1976;24(4):320-327.

Address for correspondence:

Kjell Le

Department of Electrical Engineering, University of Stavanger Stavanger, Norway

kjell.le@uis.no / trygve.eftestol@uis.no 